\title{
The Clinicopathologic Characteristics and 3-Year Survival Rates of Epithelial Ovarian Cancer in Iran During 2011-2017
}

\author{
Shahrzad Sheikh Hasani ${ }^{1}$, Mitra Modares Gilani ${ }^{1}$, Setareh Akhavan ${ }^{1}$, Azam-Sadat Mousavi ${ }^{1}$, Elham \\ Saffarieh $^{2}$, Seyedeh Reyhaneh Yousefi Sharami ${ }^{*} \mathbb{E}$
}

\begin{abstract}
Objectives: The aim of this study was to determine the 3-year overall survival among the epithelial ovarian cancer patients based on the histology, age, and the stage of the disease in Iran during 2011-2017.

Materials and Methods: This study was a cross-sectional retrospective study that was conducted on 179 newly diagnosed patients with epithelial ovarian cancer, who had referred to the gynecologic cancers clinic in a referral training hospital in Tehran during 2011-2017. The patients' data including the demographic characteristics of the patients, the stage of the disease, and the treatment type were analyzed based on the pathologic responses.

Results: Among 220 newly diagnosed patients with epithelial ovarian cancer, 179 of them were suitable for the follow-up. There were 93 death and 85 living cases among these patients and the mean age of the patients was $50.5 \pm 11.3$. In addition, most of the patients were in stage $3(60.9 \%)$ and $6.7 \%$ of them were in stage 4 . The most common pathology was serous adenocarcinoma (70.9\%). In this study, the overall survival rate had no connection with the type of tumor histology but it was related to the stage of the disease $(P=0.05)$. Finally, there was no mortality in stage one and among the mucinous adenocarcinoma cases.

Conclusions: The survival in the epithelial ovarian cancer was related to the stage of the disease and among all the pathologies, mucinous adenocarcinoma and clear cell carcinoma had the best survival rate.

Keywords: Epithelial ovarian cancer, Survival rate, Histology subtype, Age
\end{abstract}

\section{Introduction}

Ovarian cancer is the seventh most common cancer (1) and the fifth leading cause of death in the world (2). It is the main cause of death in gynecological cancers as well (3). In addition, epithelial cancer is the most prevalent ovarian cancer and usually, $60 \%$ of the patients with mild symptoms have metastasis (4). The rate of epithelial ovarian cancer increases by age growth. Environmental factors are the causes of ovarian cancer, including living in industrial countries or chemical product use, full-fat diet, personal and cultural habits, and hereditary factors (5). A decrease in ovarian cancer incidence was observed in relation to lower ovulation. This theory exists that ovary epithelial lining is sensitive to ovulatory events that can function as a cancer predisposing factor (5-7). The overall survival rate of this cancer is low because of the absence of clinical symptoms and the lack of screening since it is hard to diagnosis in the early stages (1).

In 2018, 22240 new cases of ovarian cancer were diagnosed and 14070 death cases occurred due to this type of cancer (8). Further, epithelial ovarian cancer is the eighth most common cancer in Iran and as regards mortality, it is in the 12th rank (3).
The increase in ovarian cancer incidence was reported in a study conducted in Iran. Regarding the mortality of cancer, the rate of deaths caused by cancer is an important basis for determining the ability to control cancer in different countries (2). The survival rate for ovarian cancer depends on various factors such as stage, grade, histology, age, the proper surgery treatment (residual tumor less than $1 \mathrm{~cm}$ ), and proper chemotherapy (3).

One of the main mortality causes of patients is the appropriate treatment despite the delay in referral (4) and most of the ovarian cancer cases are diagnosed in the advanced stages (60\%-74\%) $(9,10)$. Due to epidemiological changes and the growth in incidence, this study was designed to reevaluate the survival rate of epithelial ovarian cancer patients in Imam Khomeini hospital, which is the main referral center in the country.

\section{Material and Methods \\ The present study was a descriptive cross-sectional retrospective study. The patients were chosen from the epithelial ovarian cancer cases attending the Gynecologic Cancers Clinic in Imam Khomeini hospital complex, a referral training hospital in Tehran (capital of Iran). The}

Received 4 May 2018, Accepted 27 October 2018, Available online 8 December 2018

${ }^{1}$ Gynecology Oncology Department, Vali-Asr Hospital, Tehran University of Medical Sciences, Tehran, Iran. ${ }^{2}$ Abnormal Uterine Bleeding Research Center, Semnan University of Medical Sciences, Semnan, Iran. ${ }^{3}$ Department of Obstetrics and Gynaecology, Faculty of Medicine, Kurdistan University of Medical Sciences, Sanandaj, Iran.

*Corresponding Author: Seyedeh Reyhaneh Yousefi Sharami, Email: yousefi805@yahoo.com 
inclusion criteria were new cases of epithelial ovarian cancer who were examined in the hospital from April 2011 to March 2017 and the exclusion criteria were incomplete patient cases. According to intraabdominal involvement in magnetic resonance imaging (MRI), the patients with a suspicious pelvic mass in radiologic findings and carcinogenic antigen 125 (CA-125) level more than 35 were chosen for primary surgery. The patient was referred for tissue biopsy if the involvement in MRI was massive. Furthermore, the patient was treated with neoadjuvant chemotherapy and then treated by cytoreductive surgery in case of confirming the epithelial ovarian cancer. Among 220 new cases of epithelial ovarian cancer, 179 patients were suitable for the follow-up. Therefore, their demographic data including their age at the time of diagnosis, the history of malignancy, the tumor pathology (based on the international oncology staging system), the surgery stage (based on the FIGO staging), diseasefree duration, the number of recurrences, and the time between diagnosis and death were extracted and recorded. The intended histology included serous adenocarcinoma, mucinosis adenocarcinoma, endometrioid carcinoma, clear cell carcinoma, as well as other parameters such as undifferentiated carcinoma, Brenner tumors, and mixed epithelial tumor.

The stages related to the histology subtype are shown in Table 1. The follow-up of the patients was based on their visits to the clinic and it was done through phone calls if they did not refer to the center. The duration of the followup was from the time of diagnosis until the time of death and the patients who were alive after March 2017 were omitted from the study.

All data were analyzed by SPSS (version 16), and

Table 1. Demographic Data and Patient Characteristics

\begin{tabular}{|c|c|c|c|}
\hline Variables & & No. & $\%$ \\
\hline \multirow{5}{*}{ Pathology subtype } & Serous adenocarcinoma & 127 & 70.9 \\
\hline & Clear cell carcinoma & 13 & 7.3 \\
\hline & Endometrioid carcinoma & 18 & 10.1 \\
\hline & Mucinous carcinoma & 8 & 4.5 \\
\hline & Others carcinoma & 13 & 7.3 \\
\hline \multirow{3}{*}{ Tumor grade } & Low & 41 & 22.9 \\
\hline & High & 116 & 64.8 \\
\hline & Unknown & 22 & 12.3 \\
\hline \multirow{2}{*}{ Other malignancies } & No & 175 & 97.8 \\
\hline & Yes & 4 & 2.2 \\
\hline \multirow{4}{*}{ Stage } & 1 & 37 & 20.7 \\
\hline & II & 21 & 11.7 \\
\hline & III & 109 & 60.9 \\
\hline & IV & 12 & 6.7 \\
\hline \multirow{3}{*}{ Age } & $<49$ & 90 & 50.3 \\
\hline & $49-69$ & 77 & 43.0 \\
\hline & $>69$ & 12 & 6.7 \\
\hline \multirow{2}{*}{ Survival rate } & Death & 93 & 52.0 \\
\hline & Live & 86 & 48.1 \\
\hline
\end{tabular}

ANOVA, student's $t$ test, correlation, and Kaplan-Meier estimate tests were used for data analysis. $P$ value was considered less than 0.05 .

\section{Results}

Based on the results, 93 death and 85 live cases were observed among the 179 patients who were newly diagnosed with epithelial ovarian cancer undergoing the follow-up. The three-year survival for epithelial ovarian cancer was estimated at $52 \%$ in this study. Moreover, the average age for women at the time of diagnosis was $50.5 \pm 11.3$ years. However, the average age in clear cell carcinoma and endometrioid carcinoma was less than the average age for other pathologies ( 43.5 and 44.3 years, respectively). Additionally, the average time for relapse after treatment was $16.5 \pm 12.4$ months and the average time between diagnosis and death was $29.8 \pm 13.7$ months. All the patients were treated at the cancer clinic and $2 \%$ of the patients were simultaneously suffering from other malignancies as well. The CA 125 serum levels were more than $35 \mathrm{U} / \mathrm{mL}$ (the reference level) for most of the patients (81\%).

Similarly, the most common subtype histology was serous adenocarcinoma (70.9\%). Other pathologies were endometrioid carcinoma (10.1\%), clear cell carcinoma $(7.3 \%)$, and other cases such as undifferentiated adenocarcinoma, as well as Brenner tumors and mixed epithelial tumor (7.3\%) and mucinous adenocarcinoma $(4.5 \%)$, the frequency of which is displayed in Figure 1.

The longest disease-free time was 33 months and it was observed in mucinous adenocarcinoma cases. On the other hand, the shortest disease-free time was 9 months and belonged to other cases such as undifferentiated adenocarcinoma, Brenner tumors, and the mixed epithelial tumor. This difference was not statistically significant $(P=0.096)$ except for the mucinous adenocarcinoma cases in which no deaths were reported. The most and the least survival rate belonged to clear cell carcinoma with the average time of 56 months while the least survival was related to the other groups such as undifferentiated adenocarcinoma, Brenner tumors, and mixed epithelial
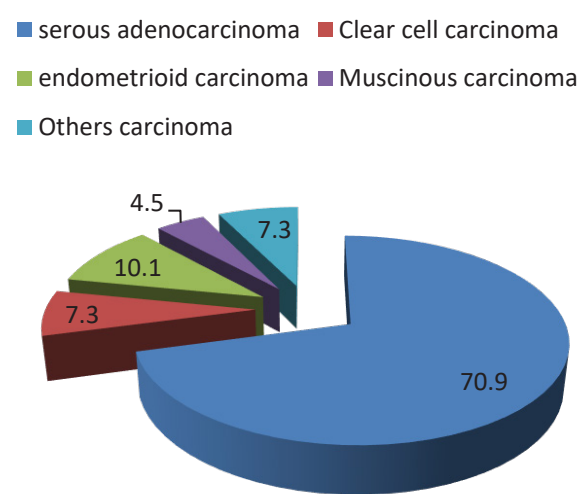

Figure 1. Pathologic Subtype Frequency of Epithelial Ovarian Cancer. 
tumor with the average time of 22.6 months. However, as shown in Figure 2, no connection was found between histology and survival $(P=0.124)$.

According to the results, $60.9 \%$ of the patients were diagnosed at stage 3 with any type of pathologies. In addition, $20.7 \%, 11.7 \%$, and $6.7 \%$ of the patients were diagnosed at stages 1,2 , and 4 , respectively. In fact, most of the patients were at advanced stages at the time of diagnosis.

In this study, the survival rate decreased with the progression of the stage and this connection was statistically significant $(P=0.05)$. No death cases were reported in stage 1 of the disease but in stage 2 , the time between diagnosis and death was reported as $37 \pm 12$ months. Further, this time was reported as $31.76 \pm 16.3$ and $21.6 \pm 6.3$ months in stages 3 and 4 , respectively. The survival rate for stage 3 was clearly better $(P=0.022)$ than the survival rate for stage 4 (Figure 3 ).

The mean age of patients suffering from serous adenocarcinoma, clear cell carcinoma, endometrioid carcinoma, mucinous adenocarcinoma, and the other tumors such as undifferentiated adenocarcinoma, Brenner tumors, and the mixed epithelial tumor was 51.76, 43.5, 44.3, 51.6, and 53.1 years, respectively. The difference between the age and pathology with the overall survival rate was significant $(P=0.013)$.

\section{Discussion}

This study aimed to determine the three-year survival rate for epithelial ovarian cancer and to investigate the relationship between tumor histology, age and the stage of the disease with the overall survival rate and was conducted on 179 patients. The three-year overall survival rate was $52 \%$. The stage of the disease and age based on the subtype pathology had a significant relationship with the survival rate but no connection was found between the histology subtype and the overall survival rate.

In a similar study conducted in Hong Kong, the overall survival rate was $69.5 \%$ (11). In another study performed in Sweden, the average survival was reported as $68.2 \%$ (12). Arab et al found that the overall survival was 59\% in Iran during 2000-2004, which is closer to our results (3). In similar research in Poland, the three-year survival was $52.2 \%$ and the average three-year survival was reported as $53.6 \%, 52.6 \%, 40.4 \%$, and $55.5 \%$ in Austria, Germany, England, and France during 2000-2007, respectively (13). This discrepancy in the survival rates can be due to the ethnic differences or various lifestyles in different countries.

In most of the studies, the association between the stage of the disease and survival was shown so that the survival rate decreased by an increase in the cancer stage $(1,6)$. In our study, the relationship between the stage of the disease and survival rate was significant $(P=0.05)$. This connection can clearly be observed in the third and fourth stages of the disease. The time interval of 31.76

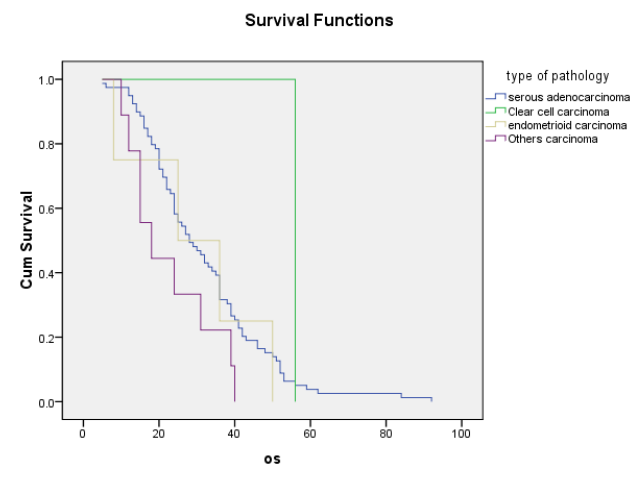

Figure 2. Relationship Between Overall Survival and Pathologic Subtype.

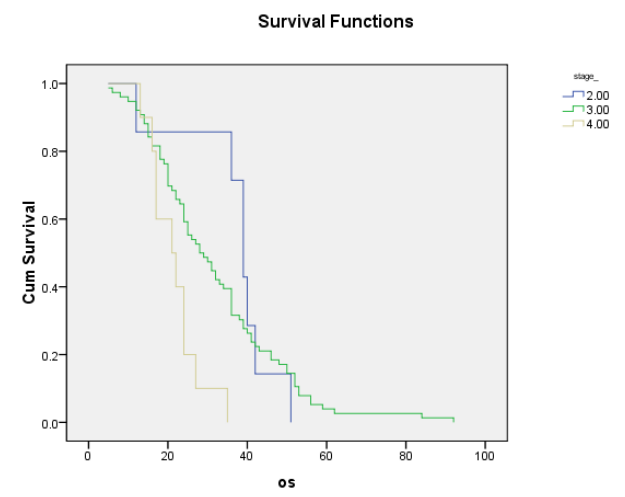

Figure 3. Relationship Between Overall Survival and the Stage of Disease.

months between the time of diagnosis and death in stage 3 in comparison with the 21.6 months in stage $4(P=0.022)$ is similar to the results of the study conducted in Taiwan in 2018 (1). This shows a decrease in the rate of survival even from stage 3 to stage 4 , both of which are considered as advanced stages and the need for diagnosing disease in earlier stages.

It is noteworthy that in our study, $32.4 \%$ of the patients were at an early stage while $67.6 \%$ of them were at an advanced stage. In a study conducted in Poland, which compared the stages of the disease in different years, it was shown that in 1990, the early-stage cases were $33.3 \%$ and this number reached $41.3 \%$ in 2015 . In addition, the advanced stage cases reduced to about $30 \%$. In other words, $18.4 \%$ of cases were undefined (13). Considering the relationship between the stage and the survival rate, early diagnosis can improve the survival rates of the patients. Referring to the gynecologic oncologist at an early stage, paying close attention to the symptoms mentioned by the patient, educating the patients about taking the symptoms seriously, along with providing education to control the risk factors such as obesity, low physical activity, unhealthy diet, and smoking can be helpful in diagnosing the disease in the early stages.

The most common subtype histology was serous adenocarcinoma (70.9\%), which was similar to the results of several studies $(3,9,10,14)$. Mucinous adenocarcinoma 
had the longest progression-free survival at 33 months whereas the shortest progression-free survival belonged to the other case groups such as undifferentiated adenocarcinoma, Brenner tumors, and the mixed epithelial tumor (at 9 months).

No death cases were reported for mucinous adenocarcinoma. Clear cell carcinoma had the highest survival rate (56 months) while the other cases such as undifferentiated adenocarcinoma, Brenner tumors, and the mixed epithelial tumor had the lowest survival rate (22.67 \pm 11.4 months) although it was not statistically significant $(P=0.166)$. In a study conducted by Wong et al (11), the highest $(87.4 \%)$ and the lowest $(40.4 \%)$ survival rates belonged to mucinous adenocarcinoma and NOS adenocarcinoma, respectively.

In another study by Ji et al, the mucinous and clear cell adenocarcinoma cases had higher survival rates, which is consistent with our findings (12).

Likewise, in a study conducted in Taiwan, the highest survival rates belonged to the mucinous and clear cell adenocarcinoma cases while the lowest survival rate was related to undifferentiated carcinomas (15). In our study, the other group included undifferentiated cases, as well.

Moreover, Arab et al indicated that the overall survival rate was the highest (76\%) for clear cell carcinoma (3). It should be mentioned that $82.7 \%$ of the serous adenocarcinoma cases were detected at advanced stages whereas $84.6 \%$ of clear cell and $87.5 \%$ of the mucinous cases were found at early stages, which may explain the better survival rate in these patients. The insignificant relationship between survival and histology in our study might be due to the low number of patients compared to the other studies.

The average age of the patients in our study was $50.5 \pm 11.3$ years. In a study conducted in Denmark, the average age of the patients was 64 years (10) but in the two studies conducted in Iran, the average age of the patients was 46.6 (3) and 53.87 (9). This confirms the assumption that the age of contracting the disease is less in Iran (14) and this can be due to the high number of younger populations in Iran.

The average age in the clear cell carcinoma and endometrioid adenocarcinoma cases were lower than that of the other pathologies (43.5 and 44.3, respectively). In a study conducted by Arab et al the patients' mean age in serous, endometrioid, mucinous, and the other cases was similar to our study (16). In the case of clear cell carcinoma, the average age of the patients in our study was less than that of the study by Arab et al. The data used in their study were from 2001-2004 and our data were obtained from more recent sources. These data might show that the age for contracting the disease has become less but further studies must be conducted on this matter.

According to Arab et al, the lower incidence in Iranian women can be related to multiparity, oral contraceptive pill use, and low-fat diets. Regarding the mortality of cancer, the rate of deaths caused by cancer is an important basis for determining the ability to control cancer in different countries (2).

Future research about 5-years and 10-years survival and finding prognostic factors to the survival rate is suggested accordingly.

Limitations of the Study

In this study, the sample size was lower than our expectation due to the lack of patients' return for follow up and their non-availability. Thus, it is recommended to perform this study in a multicentric form.

\section{Conclusions}

In the current study, there was an inverse relationship between the stage of epithelial ovarian cancer and the length of time between diagnosis and death. Mucinous adenocarcinoma and clear cell carcinoma had the highest survival rates. Finally, the survival rate had no relationship with subtype histology.

\section{Conflict of Interests}

Authors declare that they have no conflict of interests.

\section{Ethical Issues}

This study approved by Tehran university of medical sciences with an ethics code of IR.TUMS.IKHC. REC.1397.191.

\section{Financial Support}

None.

\section{References}

1. Chang LC, Huang CF, Lai MS, Shen LJ, Wu FL, Cheng WF. Prognostic factors in epithelial ovarian cancer: A population-based study. PLoS One. 2018;13(3):e0194993. doi:10.1371/journal.pone.0194993

2. Sharifian A, Pourhoseingholi MA, Norouzinia M, Vahedi M. Ovarian cancer in Iranian women, a trend analysis of mortality and incidence. Asian Pac J Cancer Prev. 2014;15(24):10787-10790. doi:10.7314/ apjcp.2014.15.24.10787

3. Arab M, Khayamzadeh M, Mohit M, et al. Survival of ovarian cancer in Iran: 2000-2004. Asian Pac J Cancer Prev. 2009;10(4):555-558.

4. Moradi Y, Jafari M, Chaichian S, et al. Trends in ovarian cancer incidence in Iran. Iran J Cancer Prev. 2016;9(6):e5452.

5. DiSaia PJ, Creasman WT, Mannel RS, McMeekin DS, Mutch DG. Clinical gynecologic oncology. Elsevier Health Sciences; 2017.

6. Mohammadbygi R, Yousefi SR, Shahghaybi S, Zandi S, Sharifi K, Gharibi F. Effects of Cabergoline administration on uterine perfusion in women with polycystic ovary syndrome. Pak J Med Sci. 2013;29(4):919-922.

7. Zamani N, Modares-Gilani M, Mirmohammadkhani M, et al. The Utility of CA 125 and HE 4 in Patients Suffering From Endometrial Cancer. Int J Womens Health Reprod Sci. 2019;7(4): In press. 
8. Torre LA, Trabert B, DeSantis CE, et al. Ovarian cancer statistics, 2018. CA Cancer J Clin. 2018;68(4):284-296. doi:10.3322/caac. 21456

9. Karimi-Zarchi M, Mortazavizadeh SM, Bashardust N, et al. The Clinicopathologic Characteristics and 5-year Survival Rate of Epithelial Ovarian Cancer in Yazd, Iran. Electron Physician. 2015;7(6):1399-1406. doi:10.14661/1399

10. Gottschau M, Mellemkjaer L, Hannibal CG, Kjaer SK. Ovarian and tubal cancer in Denmark: an update on incidence and survival. Acta Obstet Gynecol Scand. 2016;95(10):1181-1189. doi:10.1111/aogs.12948

11. Wong KH, Mang OW, Au KH, Law SC. Incidence, mortality, and survival trends of ovarian cancer in Hong Kong, 1997 to 2006: a population-based study. Hong Kong Med J. 2012;18(6):466-474.

12. Ji J, Forsti A, Sundquist J, Lenner P, Hemminki K. Survival in ovarian cancer patients by histology and family history. Acta Oncol. 2008;47(6):1133-1139. doi:10.1080/02841860701784544

13. Radziszewska AU, Karczmarek-Borowska B, Wójcik S, Kluz T. Survival rates among women with ovarian cancers diagnosed in the area of Podkarpacie province in the years 1990-2015. Contemp Oncol (Pozn). 2018;22(3):151-157. doi:10.5114/wo.2018.78935

14. Arab M, Khayamzadeh $M$, Hashemi $M$, et al. Crude and age-specific incidence rate patterns for histopathologic subtypes of ovarian cancer in Iran. Arch Iran Med. 2010;13(3):203-208.

15. Chiang YC, Chen CA, Chiang CJ, et al. Trends in incidence and survival outcome of epithelial ovarian cancer: 30-year national population-based registry in Taiwan. J Gynecol Oncol. 2013;24(4):342-351. doi:10.3802/jgo.2013.24.4.342

16. Arab $M$, Khayamzadeh $M$, Tehranian $A$, et al. Incidence rate of ovarian cancer in Iran in comparison with developed countries. Indian J Cancer. 2010;47(3):322-327. doi:10.4103/0019-509x.64721

() 2019 The Author (s); This is an open-access article distributed under the terms of the Creative Commons Attribution License (http://creativecommons.org/licenses/by/4.0), which permits unrestricted use, distribution, and reproduction in any medium, provided the original work is properly cited. 